Influence of defects and domain walls on dielectric and mechanical resonances in $\mathrm{LiNbO}_{3}$

This content has been downloaded from IOPscience. Please scroll down to see the full text.

2016 J. Phys.: Condens. Matter 28015901

(http://iopscience.iop.org/0953-8984/28/1/015901)

View the table of contents for this issue, or go to the journal homepage for more

Download details:

IP Address: 131.111.184.102

This content was downloaded on 11/12/2015 at 10:50

Please note that terms and conditions apply. 


\title{
Influence of defects and domain walls on dielectric and mechanical resonances in $\mathrm{LiNbO}_{3}$
}

\author{
Guillaume F Nataf ${ }^{1,2}$, Oktay Aktas ${ }^{3,4}$, Torsten Granzow ${ }^{1}$ \\ and Ekhard K H Salje ${ }^{1,4}$ \\ ${ }^{1}$ Materials Research and Technology Department, Luxembourg Institute of Science and Technology, \\ 41 Rue du Brill, 4422 Belvaux, Luxembourg \\ 2 SPEC, CEA, CNRS, Université Paris-Saclay, CEA Saclay, 91191 Gif sur Yvette, France \\ 3 Department of Materials, ETH Zurich, 8093 Zurich, Switzerland \\ ${ }^{4}$ Department of Earth Sciences, University of Cambridge, Cambridge, UK \\ E-mail: ekhard@esc.cam.ac.uk
}

Received 22 September 2015, revised 21 October 2015

Accepted for publication 27 October 2015

Published 7 December 2015

\begin{abstract}
Monodomain and periodically poled $\mathrm{LiNbO}_{3}$ crystals (congruent composition) show dielectric and piezoelectric resonances between $100 \mathrm{~K}$ and $900 \mathrm{~K}$. Dielectric measurements show resonances in some samples between $10-100 \mathrm{kHz}$. These resonances vanish under thermal anneal in monodomain crystals while they remain stable in periodically poled samples with high domain wall densities. The low activation energy of $0.18 \mathrm{eV}$ suggests their electronic (bi-polaronic) origin. Resonant piezoelectric spectroscopy, RPS, shows two features in virgin samples: a relaxation peak at $420 \mathrm{~K}$ and a rapid hardening when the sample was slowly heated to $\sim 500 \mathrm{~K}$. The dynamic relaxation and the hardening are related to excitations and reorientations of Li defects. The relaxations and hardening are irreversibly suppressed by high temperature anneal. We do not observe domain wall related RPS resonances in annealed samples, which excludes the existence of highly charged walls. We suggest that domain walls stabilize polaronic states with (bi-)polarons located inside or near to the ferroelectric domain walls.
\end{abstract}

Keywords: lithium niobate, domain wall, polarons

(Some figures may appear in colour only in the online journal)

\section{Introduction}

Domain walls can be functional elements of a material while the same functionality does not exist in the bulk. Typical examples are superconducting twin walls $[1,2]$, polarity and ferroelectricity in ferroelastic domain walls in $\mathrm{CaTiO}_{3}$ and $\mathrm{SrTiO}_{3}$ [3-5] and segregation of chemical species in domain walls [6]. The emerging field of domain boundary engineering Creative Commons Attribution 3.0 licence. Any further distribution of this work must maintain attribution to the author(s) and the title of the work, journal citation and DOI.
$[2,7]$ endeavors to develop such functional domain walls as device materials. A promising candidate is $\mathrm{LiNbO}_{3}$ which can show charged ferroelectric domain walls while such charges are absent in the bulk $[8,9]$. The condition for charged walls is that they are inclined with respect to the equilibrium direction [10]. They have previously been observed in $\mathrm{Pb}\left(\mathrm{Zr}, \mathrm{TiO}_{3}\right)$ thin films [11] and calculations from Eliseev et al have shown that the static conductivity drastically increases at the inclined 'head to head' domain walls even for small incline angles [12]. It has also been amply demonstrated by techniques such as electron microscopy that charged domain walls, particularly in the most extreme case of a 'head-to-head' or 'tail-to-tail' 
configuration, act as barriers for defect movement, creating regions of increased defect density [13-15]. Such inclined walls generate local strain in the bulk while walls in mechanical equilibrium are neither charged nor do they strain the lattice. Congruent $\mathrm{LiNbO}_{3}$ with non-inclined walls still contain significant defect structures [16-18] so that it can be speculated that these defects may decorate the walls and thereby generate functionality $[19,20]$. For example, the presence of defects at domain walls, and especially oxygen vacancies, is proposed to play a role in the conduction observed at the walls in $\mathrm{BiFeO}_{3}$ and $\mathrm{Pb}(\mathrm{Zr}, \mathrm{TiO})_{3}[6,21,22]$. The same mechanisms could apply for the observed photoconductivity in $\mathrm{LiNbO}_{3}$ [8]. Kinks in domain walls could also lead to local charges and may contribute to the piezoelectric response of the sample. (Self-)organized defects have recently been seen in $\mathrm{KTaO}_{3}$ where all defects are polar and act as one coherent dipole moment at sufficiently low temperatures [23]. It was the purpose of this work to explore whether $\mathrm{LiNbO}_{3}$ shows similar correlations between defects and domain boundaries.

$\mathrm{LiNbO}_{3}$ is trigonal, belonging to the $R \overline{3} c$ space group in the paraelectric phase. With the onset of ferroelectricity at $T_{\mathrm{c}}$ near $1483 \mathrm{~K}$, the structure remains trigonal, but the inversion symmetry of the system is lifted, reducing symmetry to the $\mathrm{R} 3 \mathrm{c}$ space group. $\mathrm{LiNbO}_{3}$ is hence ferroelectric but not ferroelastic below $1483 \mathrm{~K}$. Domain structures consist exclusively of $180^{\circ}$ ferroelectric walls which are-in good approximation-strain-free in thermodynamic equilibrium while local strains may originate from coupling between the polarization and secondary displacements $[24,25]$. Non-equilibrium states in uniaxial ferroelectrics $\mathrm{LiNbO}_{3}$ and $\mathrm{LiTaO}_{3}$ are commonly observed as transient features when the polarity of the samples is inverted by an applied electric field [26-29]. Furthermore, congruently grown $\mathrm{LiNbO}_{3}$ is highly non-stoichiometric. The dominant defects are $\mathrm{Nb}$-antisite defects, i.e. niobium on a lithium site $\left(\mathrm{Nb}_{\mathrm{Li}}\right)$, with a concentration of about $1 \%$. Charge compensation is usually proposed to occur by creation of lithium vacancies $V_{\mathrm{Li}}$, of which four are needed to compensate each $\mathrm{Nb}_{\mathrm{Li}}$. The actual stoichiometry of the crystal is thus $\left[\mathrm{Li}_{0.95} \mathrm{Nb}_{0.01}\right] \mathrm{NbO}_{3}[17,18]$.

We employ dielectric spectroscopy to probe the influence of defects and domain walls on the dielectric properties. Several samples showed resonance features near $10 \mathrm{kHz}$. We assume these resonances not to be due to domain wall movement since domain walls remain pinned when low voltages are applied in $\mathrm{LiNbO}_{3}$ [30]. Samples were then examined using resonance piezoelectric spectroscopy (RPS). RPS measures all mechanical vibrations which can be excited by a weak electric field in the frequency range $100 \mathrm{kHz}$ to $2 \mathrm{MHz}$. The resonances correspond to macroscopic standing strain waves, and their squared resonance frequency is proportional to the modulus of the excited mode (mainly shear waves). The temperature dependences seen in RPS are hence proportional to the temperature evolution of the effective modulus, independent of the absolute value of the resonance frequency. If polar defects exist, they are excited together with the bulk piezoelectricity and show temperature dependences which reflect the temperature evolution of the defects together with that of the bulk. Wall movements of strain-free $180^{\circ}$ walls
Table 1. Main characteristics and experiments performed on the samples.

\begin{tabular}{llll}
\hline Name & $\begin{array}{l}\text { Domain } \\
\text { period }(\mu \mathrm{m})\end{array}$ & Supplier & Experiments \\
\hline PPLNa & 20.4 & HC Photonics & Dielectric \\
PPLNb & 28 & EQ Photonics & Dielectric + RPS \\
MLN1 & Monodomain & EQ Photonics & Dielectric + RPS \\
MLN2a & Monodomain & PI-KEM & Dielectric + RPS \\
MLN2b & Monodomain & PI-KEM & Dielectric \\
MLN2c & Monodomain & PI-KEM & Dielectric \\
\hline
\end{tabular}

in $\mathrm{LiNbO}_{3}$ do not contribute to piezoelectric resonances. We argue in this paper that defect-induced piezoelectric resonances exist in most virgin samples of $\mathrm{LiNbO}_{3}$ but that these defects can be eliminated by annealing below $900 \mathrm{~K}$. We find a correlation between dielectric defect relaxations and the number of domain boundaries and conclude that domain walls stabilize and may even attract defects. We conclude that ferroelectric walls in $\mathrm{LiNbO}_{3}$ are defect-decorated and hence weakly charged.

\section{Methods}

Six samples of congruent single crystal $\mathrm{LiNbO}_{3}$ have been used for this work. A periodically poled single crystal, commercially available from HC Photonics Corps, labeled as PPLNa, had a domain period of $20.4 \mu \mathrm{m}$ (i.e. a domain wall density of $98 \mathrm{~mm}^{-1}$ ). A periodically poled single crystal with domain period $28 \mu \mathrm{m}$ (i.e. a domain wall density of $71 \mathrm{~mm}^{-1}$ ), labelled as PPLNb, and a monodomain single crystal, labelled as MLN1, were purchased from EQ Photonics GmbH. Samples MLN2a, MLN2b and MLN2c were purchased from PI-KEM Ltd and cut by ourselves from a single domain wafer. All sampled had platelet geometry with $\sim 5 \times 5 \times 0.5 \mathrm{~mm}^{3}$ dimensions and short edges along the $c$ direction. The monodomain wafers were poled by the manufacturer near the Curie temperature, without field-cooling. The poling process of the periodically poled single crystals is unknown, but conventionally done at room temperature or slightly above [31]. The characteristics of the samples are summarized in table 1.

Impedance spectroscopy was performed as a function of temperature with a Novocontrol Concept 40 dielectric spectrometer to study the evolution of the dielectric permittivity and loss. The samples were electroded with silver paste. For comparison, single measurements were also performed with sputtered gold electrodes, but since no significant effect of the electrode preparation on the results was observed, the painted silver electrodes were preferred as the less complicated preparation method. These measurements were made between $273 \mathrm{~K}$ and $473 \mathrm{~K}$ in $25 \mathrm{~K}$ steps, before RPS. They were repeated after RPS between $143 \mathrm{~K}$ and $573 \mathrm{~K}$ in $15 \mathrm{~K}$ steps on heating and cooling. The frequency range of the measurements was $1 \mathrm{~Hz}-1 \mathrm{MHz}$ with an ac-voltage of $1 \mathrm{~V}$ (rms). Each spectrum contained 240 points, logarithmically spaced. The data were analyzed using the software GRAFITY (GrafityLabs). The 

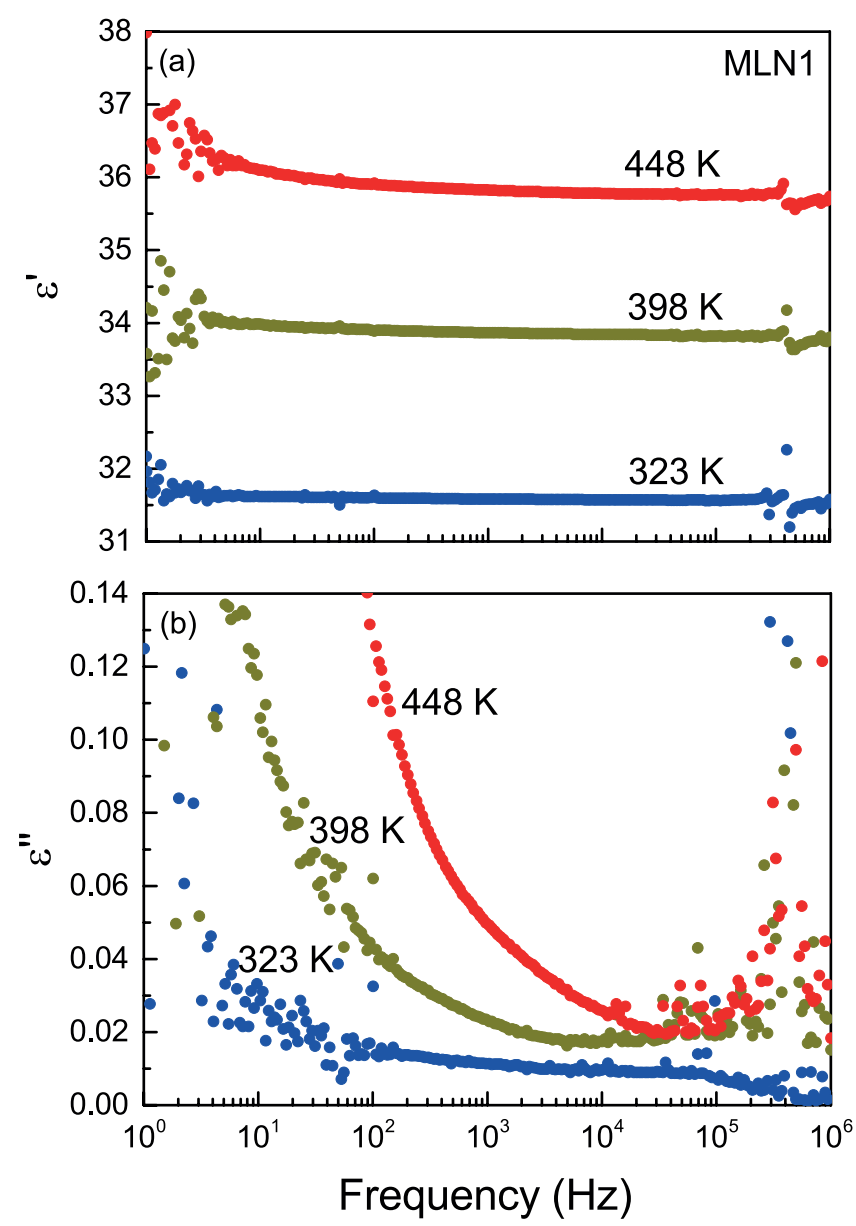

Figure 1. MLN1. (a) Real and (b) imaginary part of the permittivity as a function of the excitation frequency for temperatures between $323 \mathrm{~K}$ and $448 \mathrm{~K}$.

characteristic relaxation times of the dielectric relaxations were deduced from a fit using the Debye model.

RPS was performed between $100 \mathrm{~K}$ and $860 \mathrm{~K}$. Initially samples were inserted into an open cycled (Orange) cryostat for measurements between $100 \mathrm{~K}$ and $300 \mathrm{~K}$ performed on heating in $5 \mathrm{~K}$ steps. For these measurements an ac-voltage of $25 \mathrm{~V}$ was applied across the large surfaces of the samples using silver paste as electrodes. Each spectrum contained 65000 points between 10 and $1200 \mathrm{kHz}$. After low temperature measurements, a Netzsch furnace was used to perform RPS measurements between $300 \mathrm{~K}$ and $860 \mathrm{~K}$ in both cooling and heating sequences. These measurements were performed twice. In the first set of high temperature measurements, heating data were collected in $5 \mathrm{~K}$ steps while cooling data were collected in $10 \mathrm{~K}$ steps. In the second set, heating and cooling data were collected in $10 \mathrm{~K}$ and $20 \mathrm{~K}$ steps, respectively. Each spectrum contained 50000 points between 50 and $1200 \mathrm{kHz}$. For high temperature measurements an ac-voltage of $10 \mathrm{~V}$ was applied to the sample electrodes.

RPS data were analyzed using the software package IGOR PRO (WaveMetrics). Peak frequencies $f$ and full widths at half maxima (FWHM) $\Delta f$ of selected resonances were determined by fitting with an asymmetric Lorentzian profile. These values were used to determine mechanical damping (or, inverse mechanical quality factor) $Q^{-1}$, which can be expressed as

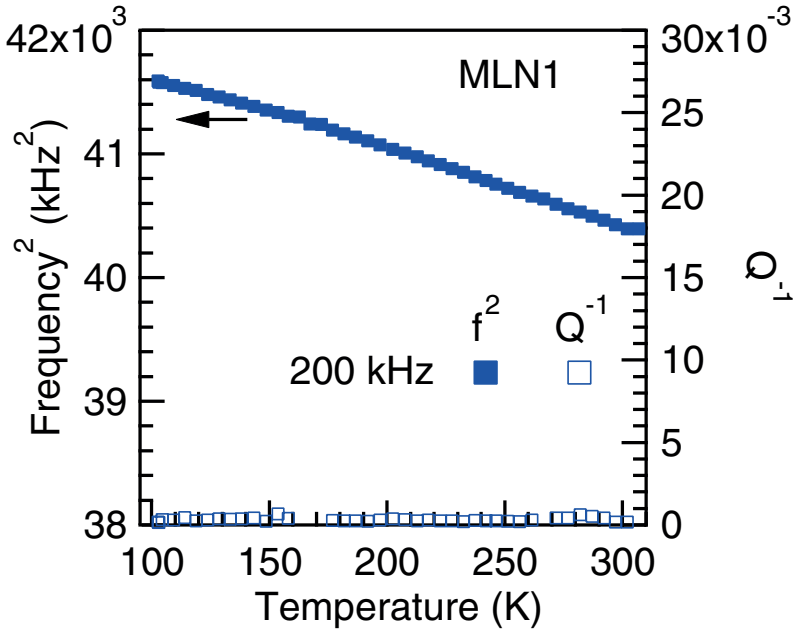

Figure 2. MLN1. Temperature evolution of squared frequency $f^{2}$ and mechanical damping $Q^{-1}$ for a resonance near $200 \mathrm{kHz}$.

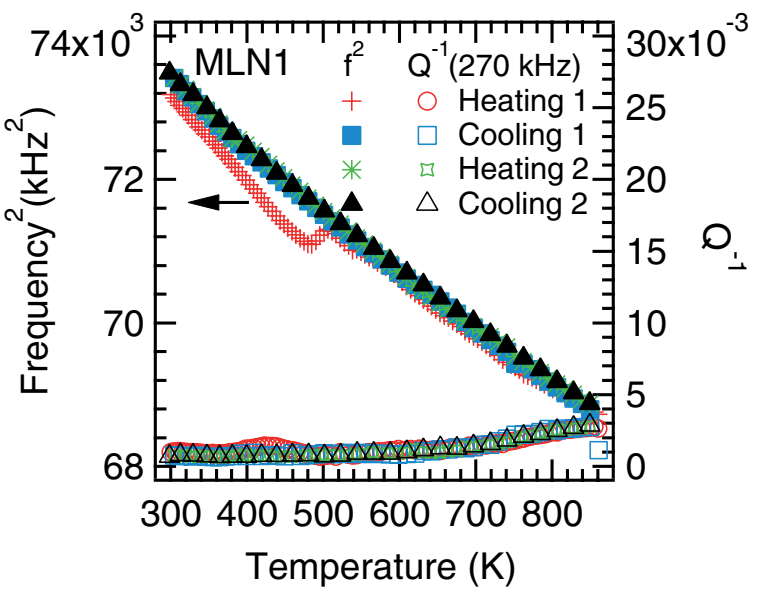

Figure 3. MLN1. Temperature evolution of the squared frequency $f^{2}$ and mechanical damping $Q^{-1}$ associated with a resonance peak near $270 \mathrm{kHz}$.

$\Delta f l f$. Since the frequency of the macroscopic standing waves depends on the dimensions, density, and orientation of the sample [32], samples with different dimensions will have different resonant frequencies. Therefore, in our analysis, we selected those resonant peaks with the highest amplitudes and those that do not interact with other resonances to obtain $Q^{-1}$ and $f$ values more accurately.

\section{Results}

\subsection{Defects in thermal equilibrium: MLN1}

Figure 1 details the temperature evolution of the permittivity in MLN1. Figure 1(a) shows the real part of the permittivity $\varepsilon^{\prime}$ and figure 1(b) the imaginary part $\varepsilon^{\prime \prime}$ at $323 \mathrm{~K}$ (blue), $398 \mathrm{~K}$ (green) and $448 \mathrm{~K}$ (red). $\varepsilon^{\prime}$ remains flat between $1 \mathrm{~Hz}$ and $10^{6} \mathrm{~Hz} . \varepsilon^{\prime \prime}$ is increasing with decreasing frequencies.

The temperature dependences of the squared frequency $f^{2}$ and mechanical damping $Q^{-1}$ of an RPS resonance in MLN1 at $200 \mathrm{kHz}$ are presented in figure 2 for the temperature range between $100 \mathrm{~K}$ and $300 \mathrm{~K}$. The squared frequency $f^{2}$ increases 

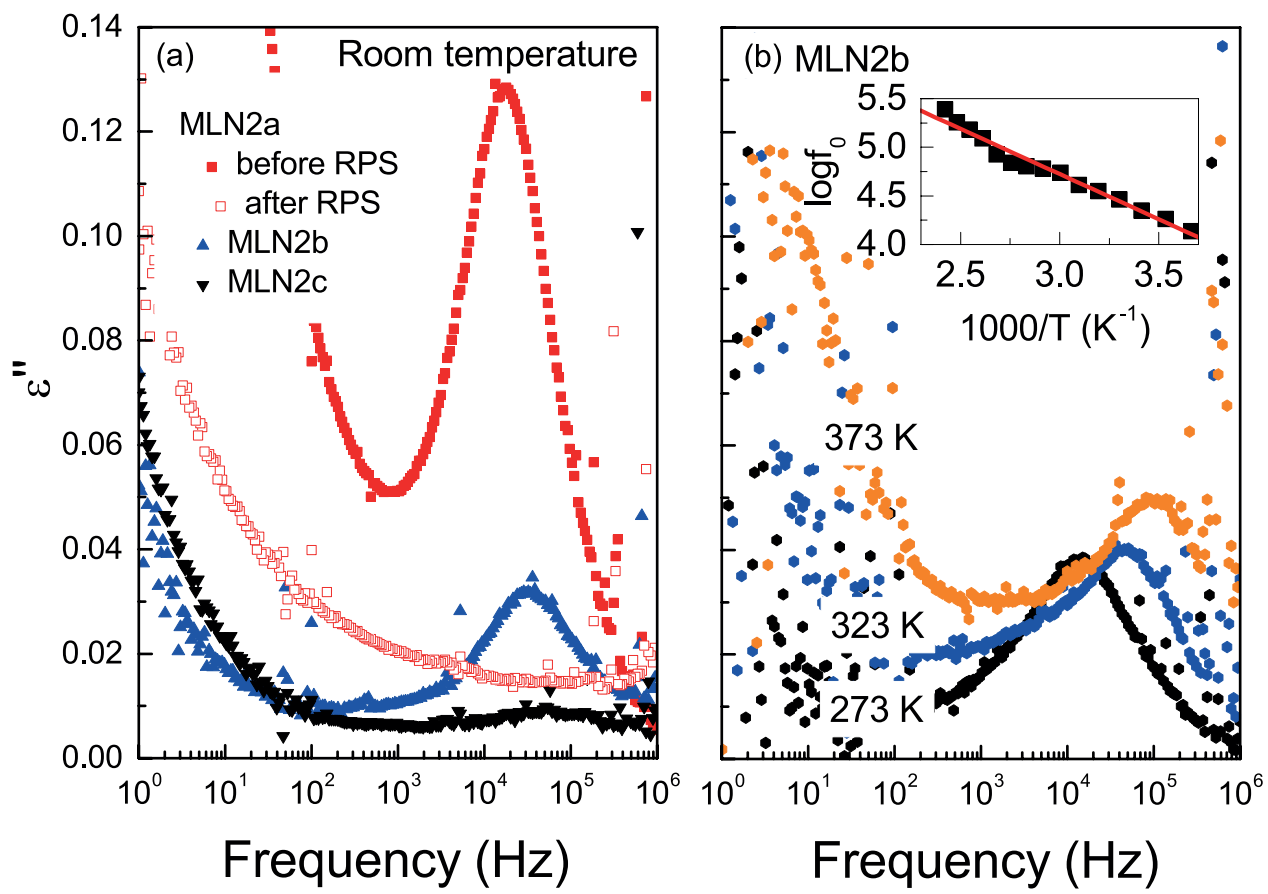

Figure 4. (a) Imaginary part of the permittivity at room temperature for MLN2a (before and after RPS), MLN2b and MLN2c. (b) Imaginary part of the permittivity at $273 \mathrm{~K}, 323 \mathrm{~K}$ and $373 \mathrm{~K}$ for MLN2b. (inset) Arrhenius fit with an activation energy of $0.18 \mathrm{eV}$.

linearly with decreasing temperature. Mechanical damping for this resonance remains low and nearly the same in the whole temperature range. The RPS measurements at low temperatures hence show the classical behavior of a defect-free sample, with stiffening on cooling.

The high-temperature RPS data of MLN1 during the first and subsequent heating and cooling runs are compared in figure 3. Data for a resonance located around $270 \mathrm{kHz}$ obtained during first heating shows a peak in the mechanical damping $\left(Q^{-1}\right)$ around $420 \mathrm{~K}$ accompanied by a step of $f^{2}$ at around $500 \mathrm{~K}$ where $Q^{-1}$ starts to deviate from the background. The two sets of measurements were performed without removing the sample from the furnace. After the first heating the peak near $420 \mathrm{~K}$ did not reoccur.

\subsection{Defect variations: $M L N 2 a, M L N 2 b$ and $M L N 2 c$}

Impedance spectroscopy of samples MLN2a, MLN2b and MLN2c shows variable behavior even though these samples have been cut from the same wafer. Figure 4(a) shows the room-temperature $\varepsilon^{\prime \prime}$ for the three samples. The red curve is the data for MLN2a. It shows a relaxation with a maximum near $30 \mathrm{kHz}$ and a large increase of $\varepsilon^{\prime \prime}$ at low frequency. After annealing during high-temperature RPS, the relaxation has disappeared and the increase at low frequency has diminished. $\varepsilon^{\prime \prime}$ for MLN2b is depicted in the blue curve. It shows a similar relaxation with a maximum near $30 \mathrm{kHz}$, even though the height of the peak is reduced. This relaxation in both MLN2a and MLN2b has a semi-circular shape on a Cole-Cole plot (not shown). The black curve shows $\varepsilon^{\prime \prime}$ for MLN2c without any relaxation peak.

Figure 4(b) shows $\varepsilon^{\prime \prime}$ for MLN2b at $273 \mathrm{~K}, 323 \mathrm{~K}$ and $373 \mathrm{~K}$. The relaxation is shifted to higher frequencies on

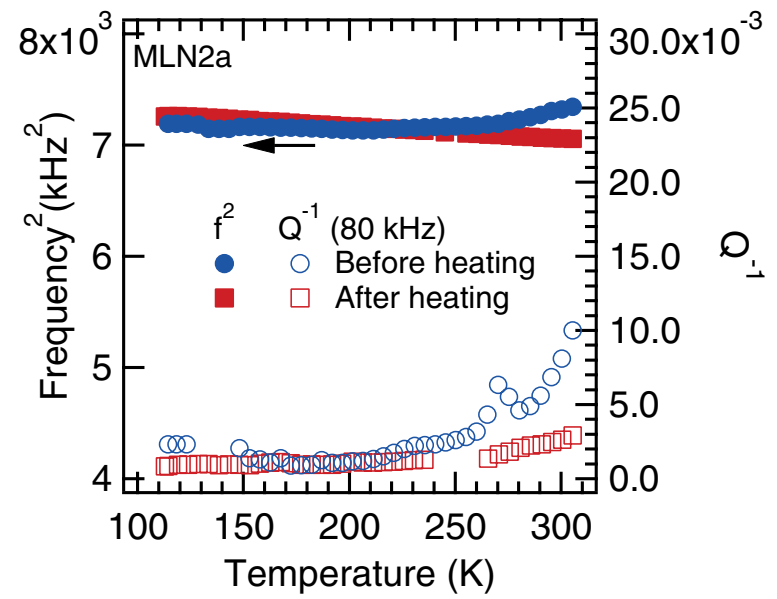

Figure 5. MLN2a. Temperature evolution for $f^{2}$ and $Q^{-1}$ for a resonance near $80 \mathrm{KHz}$ determined before and after heating the sample to $860 \mathrm{~K}$ for high temperature RPS measurements.

heating. As shown in the inset, the temperature-dependence of the relaxation frequency is well described by an Arrhenius law with an activation energy of $0.18 \mathrm{eV}$.

RPS measurements of MLN2a were performed between $100 \mathrm{~K}$ and $300 \mathrm{~K}$ and repeated after (two) high temperature RPS experiments at temperatures up to $860 \mathrm{~K}$. Values for $f^{2}$ and $Q^{-1}$ for a peak near $80 \mathrm{kHz}$ determined from these measurements are presented in figure 5 . The states before and after heating differ in $f^{2}$. Before heating to $860 \mathrm{~K}, f^{2}$ shows elastic softening at $\sim 230 \mathrm{~K}$ that vanishes after high-temperature anneal. There is also a clear reduction of $Q^{-1}$ between 250 and $300 \mathrm{~K}$ after heating compared with the data before heating.

Results from two high-temperature RPS experiments of MLN2a are compiled in figure 6. A peak in mechanical damping $Q^{-1}$ near $420 \mathrm{~K}$ is similar to that in MLN1 and is 


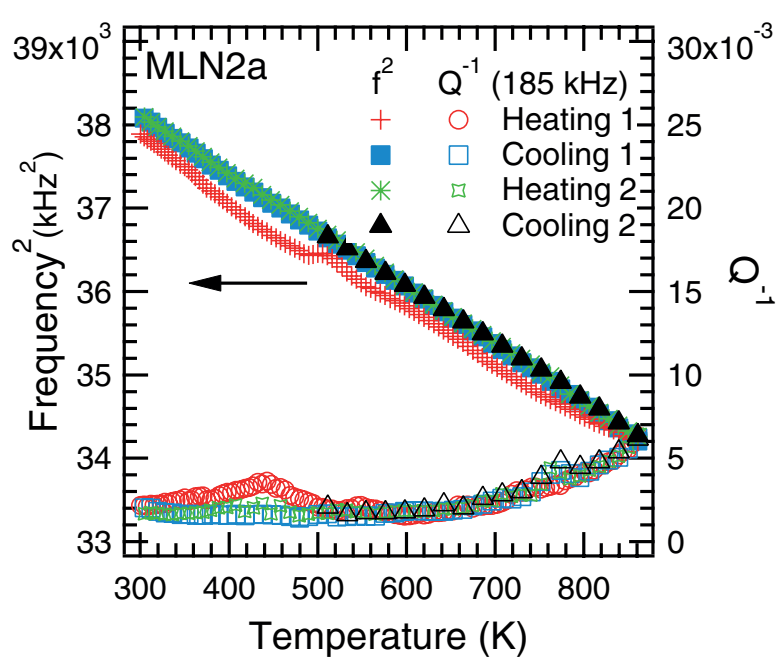

Figure 6. MLN2a. Temperature evolution of the squared frequency $f^{2}$ and mechanical damping $Q^{-1}$ associated with a resonance peak near $185 \mathrm{kHz}$.

followed by an increase of $f^{2}$ near $500 \mathrm{~K}$ in the first heating run. None of these features exist in the second experiment.

\subsection{Periodically poled lithium niobate}

Figure 7 details the temperature evolution of the permittivity in PPLNa and PPLNb. Figure 7(a) shows the real part of the permittivity at $323 \mathrm{~K}, 398 \mathrm{~K}$ and $448 \mathrm{~K}$ for data before high-temperature measurements; dielectric spectra at $323 \mathrm{~K}$, $398 \mathrm{~K}$ and $443 \mathrm{~K}$ are shown for measurements after hightemperature experiments - up to $473 \mathrm{~K}$ for PPLNa and $860 \mathrm{~K}$ for PPLNb. A change in the slope is visible near $10 \mathrm{kHz}$. Figure 7(b) shows the imaginary part of the permittivity at the same temperatures. A peak with a maximum near $10 \mathrm{kHz}$ is observed; its position is nearly temperature-independent. This peak was observed in all measurements up to $473 \mathrm{~K}$. At higher temperatures it was hidden by the increase of conductivity. A slight shift of the peak is observed between PPLNa and PPLNb. The low frequency tail depends on electrodes and is not considered any further. Figure 7(c) shows the Cole-Cole plots (imaginary versus real part of the permittivity) for PPLNa. The relaxation is the same before and after high-temperature experiments. The left end of the curves corresponds to high frequencies. The semicircles obtained were fitted with the Debye relaxation:

$$
\varepsilon=\varepsilon_{\infty}+\frac{\varepsilon_{s}-\varepsilon_{\infty}}{1+2 \mathrm{i} \pi f \tau}
$$

where $\varepsilon_{\infty}$ is the permittivity at the high frequency limit, $\varepsilon_{s}$ is the low frequency static permittivity, $\tau$ is the characteristic relaxation time of the dipoles. As shown in figure 7(c), the fit is in agreement with the experimental data.

Low temperature RPS measurements of the PPLNb sample were performed before and after heating to $860 \mathrm{~K}$. The temperature dependences of the squared frequency and mechanical damping of selected resonances are presented in figure 8 for the temperature range between $100 \mathrm{~K}$ and $300 \mathrm{~K}$. The blue symbols show that $f^{2}$ and $Q^{-1}$ decrease on cooling to $\sim 220 \mathrm{~K}$. No
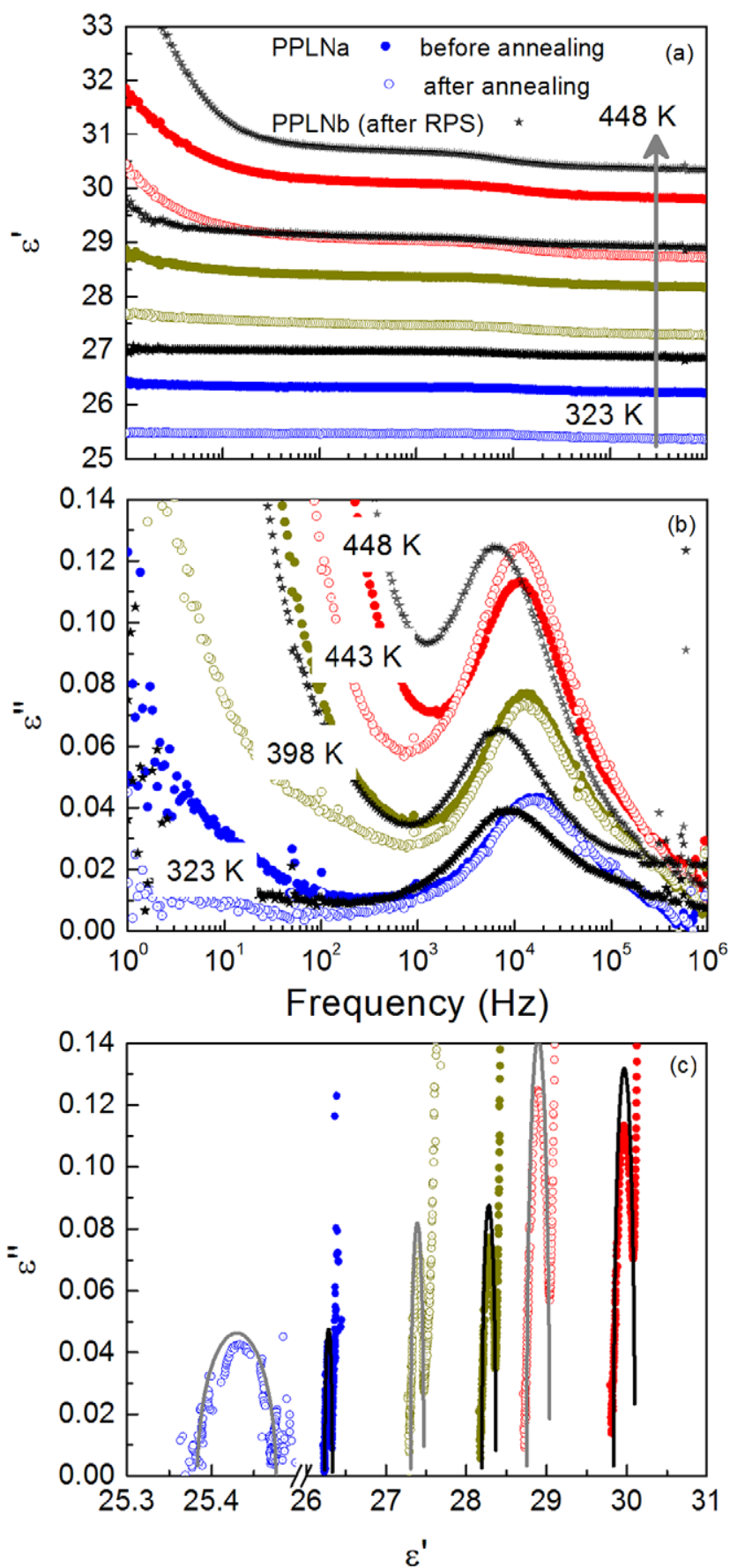

Figure 7. PPLNa and PPLNb. (a) Real part and (b) imaginary part of the permittivity as a function of the excitation frequency for temperatures between $323 \mathrm{~K}$ and $448 \mathrm{~K}$ (before and after high temperature measurements). PPLNa. (c) Cole-Cole representation. Solid lines represent the Debye fit.

phase transition has been reported for $\mathrm{LiNbO}_{3}$ in this temperature range so that softening has nothing to do with a collective transition. After (two) high temperature RPS measurements up to $860 \mathrm{~K}, f^{2}$ data do not indicate any further elastic softening on cooling (figure 8 , red symbols). There is also a clear reduction of $Q^{-1}$ between 170 and $300 \mathrm{~K}$ in comparison with earlier low temperature measurements of the un-annealed sample. However, it is worth noting that for the first measurement, 


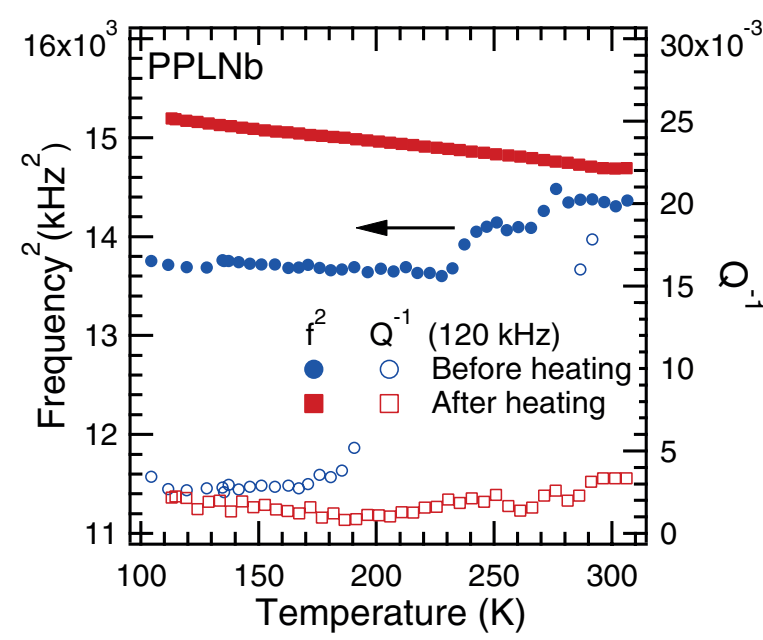

Figure 8. PPLNb. Temperature evolution for $f^{2}$ and $Q^{-1}$ for a resonance near $120 \mathrm{kHz}$ determined before and after heating the sample to $860 \mathrm{~K}$ for high-temperature RPS measurements.

between 200 and $250 \mathrm{~K}$, another resonance is interfering with the one analyzed, leading artificially to larger values of $Q^{-1}$.

RPS data of the first high temperature heating and subsequent measurements of PPLNb are compared in figure 9. Resonance frequencies near $310 \mathrm{kHz}$ of the first heating show a slight softening in $f^{2}$ with decreasing temperature and a peak in the mechanical damping $\left(Q^{-1}\right)$ around $420 \mathrm{~K}$. These effects are absent in the first cooling data (blue symbols) but a broad maximum in $f^{2}$ appears near $500 \mathrm{~K}$ (filled blue squares). Subsequent heating and cooling measurements (second set, green and black symbols) show no anomalies of the temperature evolution of the resonance frequencies and elastic damping. However, the rate of increase in $f^{2}$ for decreasing temperature is different between the four data sets, leading to a zigzag pattern in the temperature evolution of $f^{2}$ when all data sets are combined. $f^{2}$ from the first cooling measurements and that from the second heating data (green stars) do not coincide for the peak near $310 \mathrm{kHz}$ because the sample was removed from the RPS holder and remounted. Different amounts of silver paste were used in the two measurements, which could explain the discrepancy since $f^{2}$ decreases with increasing sample mass.

\section{Discussion}

A comparison of RPS damping during the first high temperature heating (red symbols in figures 3,6 and 9) of the three samples is made in figure 10 . The activation energies of $Q^{-1}$ due to a relaxation process can be calculated using [33]:

$$
Q^{-1}=Q_{m}^{-1}\left\{\cosh \left[\frac{E_{a}}{R r_{2}}\left(\frac{1}{T}-\frac{1}{T_{m}}\right)\right]\right\}^{-1}
$$

where $\mathrm{Q}_{m}^{-1}$ is the maximum of mechanical damping, $E_{a}$ is the activation energy, $R$ is the gas constant, and $r_{2}$ is the spectral width. Here $r_{2}$ is a measure of the spread of relaxation times (activation energies) involved in the process. It indicates whether the peak in $Q^{-1}$ stems from a single relaxation peak

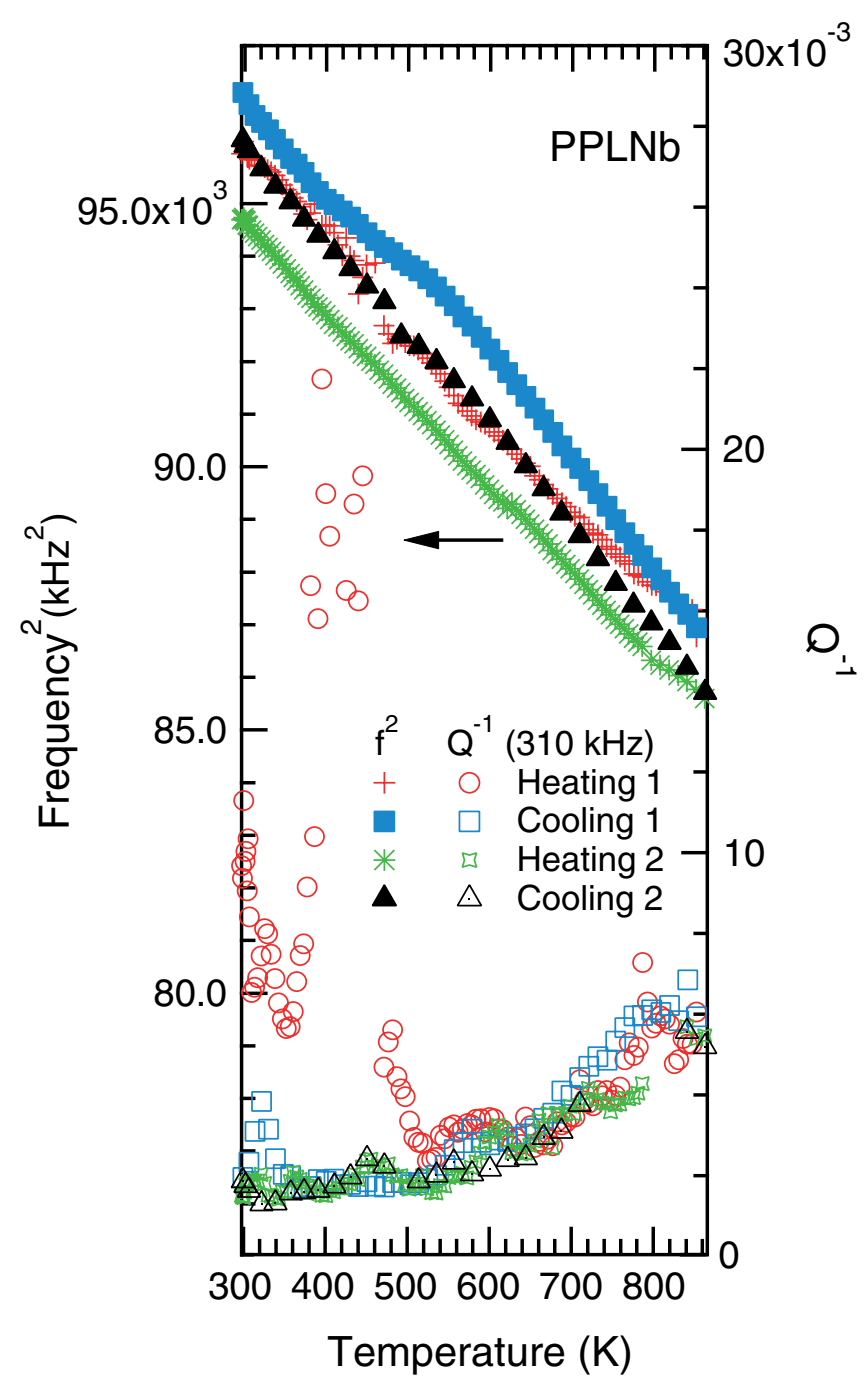

Figure 9. PPLNb. Temperature evolution of the squared frequency $f^{2}$ and mechanical damping $Q^{-1}$ associated with a resonance peak near $310 \mathrm{kHz}$.

$\left(r_{2}=1\right)$ or the product of several overlapping peaks with multiple relaxations at different frequencies and temperatures. The activation energies are found to be in the range $0.5-1 \mathrm{eV}$.

The resonance peak near $420 \mathrm{~K}$ is complemented by an increase of the moduli near $500 \mathrm{~K}$. This hardening of the shear modulus is irreversible and leads to values which characterize monodomain samples under heating and cooling conditions. Following the interpretation of Gopalan and Gupta [27] of rapid polarization changes in $\mathrm{LiTaO}_{3}$ we assume that $\mathrm{LiNbO}_{3}$ equally orients its local polar defects along the dominant polarization direction near $500 \mathrm{~K}$. Once these local dipoles are aligned they no longer change in monodomain samples. This alignment is preceded at $420 \mathrm{~K}$ by the weak relaxation peak where local dipoles resonate with the weak electric field and give rise to damping of the piezoelectric response. This effect is very strong in the periodically poled sample $\left(Q^{-1}=22 \times 10^{-3}\right)$ while the effect is one order of magnitude smaller in samples without domain wall structure. We can conclude that domain walls enhance this resonance while thermal anneal still destroys it. 
When the high temperature RPS measurements are repeated, the elastic relaxation disappears, indicating that annealing of the samples resulted in the settling or redistribution of defects. In PPLNb we found some very small variations in $f^{2}$. This results mirrors recent work by Biancoli et al [34] on solid solutions of $\mathrm{BaTiO}_{3}$ and $\mathrm{SrTiO}_{3}$. They found that although thermally stimulated current measurements do not show evidence of space charges when the sample was cooled immediately after heating, if the measurements are repeated after several days, these anomalies re-establish themselves, hence reinforcing the built-in polarization due to a preferred orientation of dipoles even in polycrystalline ceramics. Because the second set of heating and cooling measurements on PPLNb were performed approximately one week after the first set of RPS measurements in heating and cooling cycles, it is likely that a few polar dipoles were reoriented during that time interval.

Dielectric measurements on MLN1 prove that it is possible to obtain a relaxation-free spectrum from a monodomain sample with intrinsic defects of congruently grown $\mathrm{LiNbO}_{3}$. This is in agreement with previous dielectric measurements $[35,36]$. Samples MLN2a and MLN2b are also monodomain samples but show Debye relaxations near $10 \mathrm{kHz}$ which are very similar to that of the PPLN. These relaxations vanish after high temperature treatment (figure 4). Optical microscopy did not reveal domain walls and PFM measurements at various sites across the sample surface were compatible with the monodomain state. It is not excluded that antiparallel domains exist, but their volume fraction is far too low to account for the observed dielectric relaxation. In the PPLN sample, the intrinsic defects are stabilized against thermal anneal by domain walls while in monodomain MLN2a and MLN2b this stabilization is absent and the defect-induced relaxation is destroyed by heating. A possible explanation is the compensation of local charged defects by migration of intrinsic defects in congruent $\mathrm{LiNbO}_{3}$ such as lithium vacancies, which become mobile already at moderate temperatures around $420 \mathrm{~K}$ [37]. This also explains the absence of the dielectric relaxation in MLN2c: the chemical composition is identical to MLN2a and MLN2b, which have been cut from the same wafer. However, the fragile local defect structure that causes the dielectric relaxation may have been altered in MLN2c, e.g. by uncontrolled heating during machining. Influence of annealing on defects and dielectric properties had already been observed in iron-doped [36] and magnesiumdoped lithium niobate [38].

The main result is hence that the permittivity of PPLN shows a strong Debye relaxation with a maximum near $10 \mathrm{kHz}$ (figure 7) that does not change when the sample is annealed at $860 \mathrm{~K}$. The relaxation is unlikely to stem from the motion of the domain walls themselves which would lead to much more complex relaxation pattern. Furthermore, the coercive field in $\mathrm{LiNbO}_{3}$ is very large and domain walls are effectively pinned at the electric field strength employed in this study [30]. The dielectric relaxation in PPLN may be electronic or ionic in origin. However, since all the samples are of congruent composition, variations in the stoichiometry between samples cannot account for the observed relaxation. Two observations

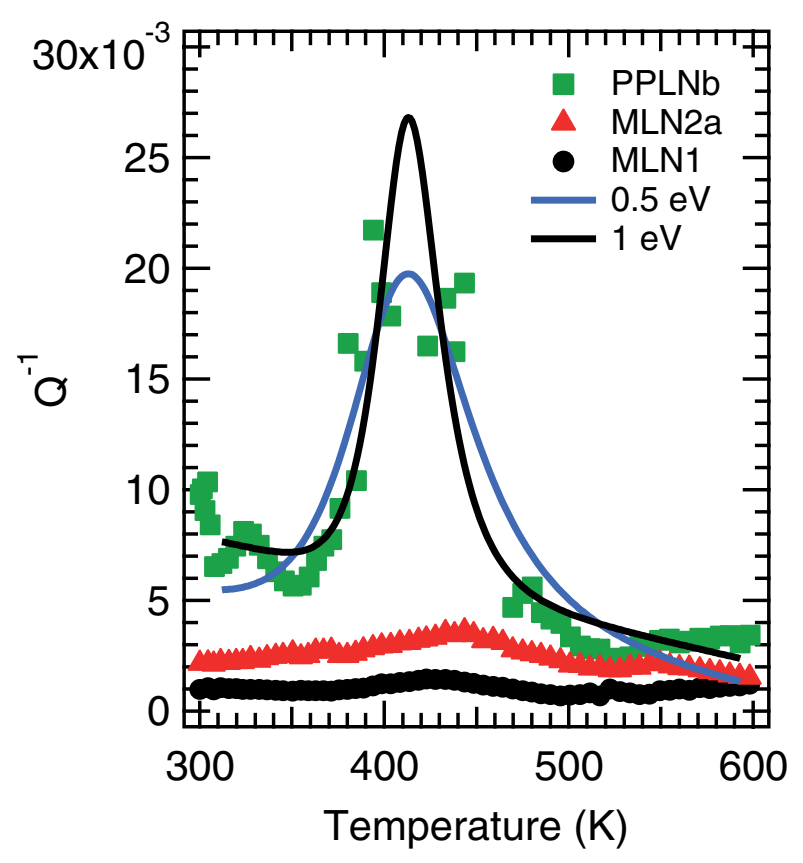

Figure 10. Mechanical damping in PPLNb, MLN1, and MLN2a samples obtained during the first high temperature measurements in heating sequences (presented in figures 3,6 and 9). The blue and black lines correspond to a fit with an activation energy of $0.5 \mathrm{eV}$ and $1 \mathrm{eV}$, respectively.

confirm this point: (i) the relaxation is observed in two PPLN from different suppliers (ii) MLN1 has a relaxation-free spectrum, while it comes from the same supplier as PPLNb. The different domain wall densities in PPLNa and PPLNb did not have any apparent influence on the dielectric behavior; the slightly higher permittivity for the sample with fewer domain walls is within the error margin of the experiment. It is worth noting that the small shift in the imaginary part of the permittivity between PPLNa and PPLNb might be due to minuscule changes in composition or defect structure. We can compare the behavior of $\mathrm{LiNbO}_{3}$ with highly twinned leucite [39] where small movements of ions were correlated within individual domain sizes. Experimentally, the dielectric relaxation in leucite shows a frequency maximum which changes with changing size of the domains. While it is tempting to explain the dielectric resonance peak in PPLNb similarly, we find that the activation energy is very small, $0.18 \mathrm{eV}$. This value is smaller than the energies needed for a long range motion of ions in lithium niobate: ionic conductivity of lithium, hydrogen or oxygen vacancies is associated with much larger activation energies of $1-1.25 \mathrm{eV}[37,40,41]$. The origin of the relaxation is therefore likely to be of electronic nature. It would be compatible with the bi-polaron energy of $E=0.27 \mathrm{eV}$ discussed by Schirmer et al [42] provided that the hopping energy is $E / 2$, as usually assumed for (bi-)polarons. In addition, it is possible that MLN2a and MLN2b contain ionic defects induced by the congruent composition, but that these defects are in a fixed non-equilibrium configuration after high-temperature poling and do no longer contribute to the dielectric relaxation after anneal. This agrees with the observation that the singledomain sample MNL1 shows no dielectric resonance, nor any 
piezoelectric anomaly, despite the Li deficiency of the congruently melting sample.

\section{A simple model}

The dielectric relaxation both in monodomain and periodically poled $\mathrm{LiNbO}_{3}$ arises from non-equilibrium charged polaronic states such as $\mathrm{Nb}_{\mathrm{Li}}^{4+}$ polarons. Annealing single domain samples leads to a rearrangement of the defect structure, particularly the $\mathrm{Li}$ vacancies, that causes the relaxation to vanish. In the PPLN sample, ferroelectric $180^{\circ}$ domain walls stabilize the charged defect structures. The charged states apparently do not contribute to dc conductivity [8]. The question is how the nominally uncharged and unstrained domain walls can stabilize the defect structure: the walls are not inclined [10] and do not share the high concentration of carriers as seen in transient domain states before thermal anneal. Heating a sample eliminates most defects and increases the shear modulus of the sample while the wall-related dielectric anomaly persists. It is likely, therefore, that the charged defects are located at or near ferroelectric domain walls. We can now speculate which geometrical domain wall pattern would generate such defects. Experimentally the overall orientation of the walls is parallel to the crystallographic $c$-axis. This does not mean that local wall bending is impossible, in particular if the domain walls are some unit cells wide. Similar geometries are observed on $\mathrm{LiNbO}_{3}$ surfaces when domain walls are pinned at defects $[43,44]$. Such 'wide' walls could meander in the matrix whereby each bending would lead to a head-to-head or tail-totail configuration of the dipoles in the adjacent matrix. Such bends would be energetically favorable in positions where the resulting bound charges can be locally compensated, i.e. at positions where charges already exists in the form of charged polaronic states. The polaronic state and the local deformation of the domain wall would mutually stabilize one another. Following this idea we anticipate that ferroelectric walls in $\mathrm{LiNbO}_{3}$ are decorated and weakly charged. We now compare dielectric resonances in periodically poled samples with the transient resonances in monodomain samples and find that both have the same relaxation frequency. The monodomain defects are commonly believed to be related to charge $\mathrm{Nb}$-antisites where the defect dipole can be aligned with the spontaneous polarization at moderate temperatures [37]. It is possible, therefore, that the charged walls contain a similar local defect configuration. This would mean that bending a domain wall in $\mathrm{LiNbO}_{3}$ would imply the generation of such polar, topological point defects including the switch of $\mathrm{Li}$ positions. This stabilization effect of the domain walls is also visible in the lack of temperature dependence of the relaxation frequency in the PPLN sample. The fundamental relaxation frequency in the monodomain and polydomain samples is very similar, but the relaxation frequency in the first one increases slightly with increasing temperature, resulting in an activation energy of $0.18 \mathrm{eV}$. In the PPLN sample, there is no such temperature dependence. Further work using highresolution transmission electron microscopy is highly desirable to clarify the proposed model.

\section{Acknowledgments}

GFN and TG are grateful to National Research Fund (Luxembourg) for support (FNR/P12/4853155/Kreisel). EKHS is grateful to EPSRC for support (EP/K009702/1).

\section{References}

[1] Kim Y, Alexe M and Salje E K H 2010 Nanoscale properties of thin twin walls and surface layers in piezoelectric $\mathrm{WO}_{3-\mathrm{x}}$ Appl. Phys. Lett. 96032904

[2] Salje E K H 2010 Multiferroic domain boundaries as active memory devices: trajectories towards domain boundary engineering ChemPhysChem 11 940-50

[3] Yokota H, Usami H, Haumont R, Hicher P, Kaneshiro J, Salje E K H and Uesu Y 2014 Direct evidence of polar nature of ferroelastic twin boundaries in $\mathrm{CaTiO}_{3}$ obtained by second harmonic generation microscope Phys. Rev. B 89144109

[4] Van Aert S, Turner S, Delville R, Schryvers D, Van Tendeloo G and Salje E K H 2012 Direct observation of ferrielectricity at ferroelastic domain boundaries in $\mathrm{CaTiO}_{3}$ by electron microscopy Adv. Mater. 24 523-7

[5] Salje E K H, Aktas O, Carpenter M A, Laguta V V and Scott J F 2013 Domains within domains and walls within walls: evidence for polar domains in cryogenic $\mathrm{SrTiO}_{3}$ Phys. Rev. Lett. 111247603

[6] Seidel J et al 2010 Domain wall conductivity in La-Doped $\mathrm{BiFeO}_{3}$ Phys. Rev. Lett. 105197603

[7] Catalan G, Seidel J, Ramesh R and Scott J F 2012 Domain wall nanoelectronics Rev. Mod. Phys. 84 119-56

[8] Schröder M, Haußmann A, Thiessen A, Soergel E, Woike T and Eng L M 2012 Conducting domain walls in lithium niobate single crystals Adv. Funct. Mater. 22 3936-44

[9] Schröder M, Chen X, Haußmann A, Thiessen A, Poppe J, Bonnell D A and Eng L M 2014 Nanoscale and macroscopic electrical ac transport along conductive domain walls in lithium niobate single crystals Mater. Res. Express 1035012

[10] Kämpfe T, Reichenbach P, Schröder M, Haußmann A, Eng L M, Woike T and Soergel E 2014 Optical 3D profiling of charged domain walls in ferroelectrics by Cherenkov second-harmonic generation Phys. Rev. B 89035314

[11] Jia C-L, Mi S-B, Urban K, Vrejoiu I, Alexe M and Hesse D 2008 Atomic-scale study of electric dipoles near charged and uncharged domain walls in ferroelectric films Nat. Mater. 7 57-61

[12] Eliseev E A, Morozovska A N, Svechnikov G S, Gopalan V and Shur V Y 2011 Static conductivity of charged domain walls in uniaxial ferroelectric semiconductors Phys. Rev. B 83235313

[13] Aristov V and Kokhanchik L 1992 Scanning electron microscopy investigation of lithium niobate properties Ferroelectrics 126 353-8

[14] Aristov V V, Kokhanchik L S and Voronovskii Y I 1984 Voltage contrast of ferroelectric domains of lithium niobate in SEM Phys. Status Solidi 86 133-41

[15] Kokhanchik L S 2009 The use of surface charging in the SEM for lithium niobate domain structure investigation Micron 40 41-5

[16] Xu H, Lee D, He J, Sinnott S B, Gopalan V, Dierolf V and Phillpot S R 2008 Stability of intrinsic defects and defect clusters in $\mathrm{LiNbO}_{3}$ from density functional theory calculations Phys. Rev. B 78174103

[17] Abrahams S C and Marsh P 1986 Defect structure dependence on composition in lithium niobate Acta Crystallogr. B 42 61-8 
[18] Lerner P, Legras C and Dumas J P 1968 Stoechiométrie des monocristaux de métaniobate de lithium J. Cryst. Growth 3-4 231-5

[19] Xu H, Lee D, Sinnott S B, Gopalan V, Dierolf V and Phillpot S R 2010 Interactions of defects and domain walls in $\mathrm{LiNbO}_{3}$-insights from simulations IOP Conf. Series: Materials Science and Engineering 15012003

[20] Stone G, Lee D, Xu H, Phillpot S R and Dierolf V 2013 Local probing of the interaction between intrinsic defects and ferroelectric domain walls in lithium niobate Appl. Phys. Lett. 102042905

[21] Farokhipoor S and Noheda B 2011 Conduction through $71^{\circ}$ domain walls in $\mathrm{BiFeO}_{3}$ thin films Phys. Rev. Lett. 107127601

[22] Guyonnet J, Gaponenko I, Gariglio S and Paruch P 2011 Conduction at domain walls in insulating $\mathrm{Pb}\left(\mathrm{Zr}_{0.2} \mathrm{Ti}_{0.8}\right) \mathrm{O}_{3}$ thin films Adv. Mater. 23 5377-82

[23] Aktas O, Crossley S, Carpenter M A and Salje E K H 2014 Polar correlations and defect-induced ferroelectricity in cryogenic $\mathrm{KTaO}_{3}$ Phys. Rev. B 90165309

[24] Shapovalov K, Yudin P V, Tagantsev A K, Eliseev E A, Morozovska A N and Setter N 2014 Elastic coupling between nonferroelastic domain walls Phys. Rev. Lett. 113207601

[25] Conti S, Müller S, Poliakovsky A and Salje E K H 2011 Coupling of order parameters, chirality, and interfacial structures in multiferroic materials. J. Phys.: Condens. Matter 23142203

[26] Gopalan V and Gupta M C 1996 Origin of internal field and visualization of $180^{\circ}$ domains in congruent $\mathrm{LiTaO}_{3}$ crystals J. Appl. Phys. 806099

[27] Gopalan V and Gupta M C 1996 Observation of internal field in $\mathrm{LiTaO}_{3}$ single crystals: Its origin and time-temperature dependence Appl. Phys. Lett. 68888

[28] Paturzo M, Alfieri D, Grilli S, Ferraro P, De Natale P, de Angelis M, De Nicola S, Fińizio A and Pierattini G 2004 Investigation of electric internal field in congruent $\mathrm{LiNbO}_{3}$ by electro-optic effect Appl. Phys. Lett. 855652

[29] Paturzo M et al 2005 On the origin of internal field in Lithium Niobate crystals directly observed by digital holography Opt. Express 135416

[30] Jach T, Kim S, Gopalan V, Durbin S and Bright D 2004 Long-range strains and the effects of applied field at $180^{\circ}$ ferroelectric domain walls in lithium niobate Phys. Rev. B 69064113
[31] Myers L E, Eckardt R C, Fejer M M, Byer R L, Bosenberg W R and Pierce J W 1995 Quasi-phase-matched optical parametric oscillators in bulk periodically poled $\mathrm{LiNbO}_{3}$, J. Opt. Soc. Am. B 122102

[32] Migliori A and Sarrao J 1997 Resonant Ultrasound Spectroscopy (New York: Wiley)

[33] Weller M, Li G Y, Zhang J X, Kê T S and Diehl J 1981 Accurate determination of activation enthalpies associated with the stress-induced migration of oxygen or nitrogen in tantalum and niobium Acta Metall. 29 1047-54

[34] Biancoli A, Fancher C M, Jones J L and Damjanovic D 2015 Breaking of macroscopic centric symmetry in paraelectric phases of ferroelectric materials and implications for flexoelectricity Nat. Mater. 14 224-9

[35] Mansingh A and Dhar A 1985 The ac conductivity and dielectric constant of lithium niobate single crystals J. Phys. D.: Appl. Phys. 18 2059-71

[36] Barbosa P C, de Paiva J A C, Filho J M, Hernandes A C, Andreeta J P and Sombra A S B 1991 Dielectric relaxation process and pyroelectric currents in $\mathrm{LiNbO}_{3}: \mathrm{Fe}$ single crystals Phys. Status Solidi 125 723-9

[37] Gopalan V, Dierolf V and Scrymgeour D A 2007 Defectdomain wall interactions in trigonal ferroelectrics Anпи. Rev. Mater. Res. 37 449-89

[38] Meyer N, Nataf G F and Granzow T 2014 Field induced modification of defect complexes in magnesium-doped lithium niobate J. Appl. Phys. 116244102

[39] Palmer D and Salje E H 1990 Phase transitions in leucite: Dielectric properties and transition mechanism Phys. Chem. Miner. 17 444-52

[40] Hüger E, Rahn J, Stahn J, Geue T, Heitjans P and Schmidt H 2014 Lithium diffusion in congruent $\mathrm{LiNbO}_{3}$ single crystals at low temperatures probed by neutron reflectometry. Phys. Chem. Chem. Phys. 16 3670-4

[41] Birnie D P 1993 Analysis of diffusion in lithium niobate J. Mater. Sci. 28 302-15

[42] Schirmer O F, Imlau M, Merschjann C and Schoke B 2009 Electron small polarons and bipolarons in $\mathrm{LiNbO}_{3}$ J. Phys.: Condens. Matter 21123201

[43] Yang T J, Gopalan V, Swart P J and Mohideen U 1999 Direct observation of pinning and bowing of a single ferroelectric domain wall Phys. Rev. Lett. 82 4106-9

[44] Yan W et al 2006 The electrostatic depinning mechanism of domain wall for near-stoichiometric lithium niobate crystals J. Phys. D.: Appl. Phys. 39 4245-9 